\title{
Covid-19 e sua relação com a hipertensão arterial sistêmica: uma revisão bibliográfica
}

Covid-19 and its relationship with systemic arterial hypertension: a literature review

Covid-19 y su relación con la hipertensión arterial sistémica: una revisión de la literatura

Rayanne Lima Lopes ${ }^{1 *}$, Lília Garcia Leite Falcão², Caroline de Oliveira Leão ${ }^{2}$ Érica Karen Sousa Novais $^{2}$, Caio Oslo Oliveira Cardoso ${ }^{2}$, Luna Marques Macedo², Maíra Caroline Silva Rodrigues², Victoria Maria Alves Rego², Ellen Beatriz Teixeira Dias², Maristella Mares Leite Cirilo Moura'.

\section{RESUMO}

Objetivo: Compreender a relação entre a covid-19 e a hipertensão arterial sistêmica. Revisão bibliográfica: O coronavírus adentra nas células por meio do receptor da ECA II. Paralelamente, pacientes portadores da HAS apresentam esses receptores em maior quantidade. Desse modo, estariam mais vulneráveis ao avanço do vírus nas células extrapulmonares. Assim, foi observado o aumento da morbidade e mortalidade da covid19 em pacientes com HAS. A maioria dos óbitos e complicações são em pacientes com 60 anos ou mais que apresentam alguma comorbidade. Além disso, é importante entender qual a correlação entre a covid-19 e a HAS e porque essa comorbidade gera um pior prognóstico em pacientes infectados pelo coronavírus. Primeiro, para que sejam mais efetivos comportamentos que previnam a contaminação nesse grupo de risco, bem como otimizar a terapêutica da hipertensão a fim de evitar complicações na saúde dos pacientes. Considerações finais: A hipertensão arterial tem associação significativa com a gravidade e mortalidade da covid-19. Os pacientes hipertensos e infectados pelo coronavírus podem apresentar severas manifestações clinicas e complicações em órgãos-alvos.

Palavras-chave: Covid-19, Saúde, Hipertensão arterial sistêmica.

\begin{abstract}
Objective: To understand the relationship between covid-19 and systemic arterial hypertension. Review bibliographic: Coronavirus enters cells through the ACE II receptor. At the same time, patients with SAH have these receptors in greater quantity. Thus, they would be more vulnerable to the advance of the virus in extrapulmonary cells. Thus, an increase in covid-19 morbidity and mortality was observed in patients with SAH. Most deaths and complications are in patients aged 60 years or older who have some comorbidity. In addition, it is important to understand the correlation between covid-19 and SAH and why this comorbidity generates a worse prognosis in patients infected with coronavirus. First, so that behaviors that prevent contamination in this risk group are more effective, as well as to optimize the treatment of hypertension in order to avoid complications in the health of patients. Final considerations: Hypertension has a significant association with covid-19 severity and mortality. Hypertensive and coronavirus-infected patients may present with severe clinical manifestations and complications in target organs.
\end{abstract}

Keywords: Covid-19, Health, Systemic arterial hypertension.

\section{RESUMEN}

Objetivo: Este constructo tuvo como objetivo comprender la relación entre covid-19 y la hipertensión arterial sistémica. Revisión bibliográfica: El coronavirus ingresa a las células a través del receptor ACE II. Al mismo tiempo, los pacientes con HSA tienen estos receptores en mayor cantidad. Por tanto, serían más vulnerables al avance del virus en las células extrapulmonares. Por tanto, se observó un aumento de la morbilidad y la mortalidad de covid-19 en pacientes con HSA. La mayoría de las muertes y complicaciones ocurren en pacientes de 60 años o más que tienen alguna comorbilidad. Además, es importante comprender la

\footnotetext{
${ }^{1}$ Faculdade Santo Agostinho (FASA), Vitória da Conquista - BA. *E-mail: rayannelimagbi@gmail.com

2 Faculdade Santo Agostinho de Itabuna (FASAI), Itabuna - BA.
} 
correlación entre covid-19 y HAS y por qué esta comorbilidad genera un peor pronóstico en pacientes infectados por coronavirus. Primero, hacer más efectivas las conductas que previenen la contaminación en este grupo de riesgo, así como optimizar el tratamiento de la hipertensión para evitar complicaciones en la salud de los pacientes. Consideraciones finales: Se pudo concluir que la hipertensión arterial se asocia significativamente con la gravedad y mortalidad del covid-19. Los pacientes hipertensos e infectados por coronavirus pueden presentar manifestaciones clínicas graves y complicaciones en los órganos diana.

Palabras clave: Covid-19, Salud, Hipertensión arterial sistémica.

\section{INTRODUÇÃO}

O ano de 2020 foi marcado por uma pandemia, decretada pela Organização Mundial da Saúde (OMS) em 11 de março de 2020, causada pelo coronavírus da síndrome respiratória grave (SARS-CoV-2). Este, pertence a linhagem $B$ dos betas coronavírus e está intimamente relacionado ao vírus SARS-CoV (VELAVAN TP e MEYER CG, 2020).

A origem, a localização e o reservatório natural do SARS-CoV-2 permanecem enigmáticos, apesar de que se acredite que o vírus seja zoonótico e os morcegos possam ser os responsáveis por causa da identidade de sequência com morcego-CoV (WU Y, et al., 2020).

O surto da covid-19, foi considerada uma emergência mundial, devido a sua rápida disseminação e alta taxa de mortalidade. As evidências mostram que os padrões imunológicos estão associados ao crescimento da doença em pacientes contaminados com o vírus. Pacientes com síndrome respiratória aguda apresentam uma diminuição nos subconjuntos de células T periféricas. Já nos pacientes recuperados, existe uma rápida restauração de subconjuntos de células T periféricas. Dessa forma, o número de células T periféricas podem servir como método diagnóstico precisa para a SARS (YANG G, et al., 2020).

O período de incubação da doença é inconstante conforme a idade do indivíduo, variando de 6 a 7 dias. Algumas pessoas podem ser assintomáticas durante esse período, porém é altamente contagioso durante o período latente. A disseminação do vírus pode ocorrer através da tosse ou espirro, ou superfícies contaminadas (LIMA AR, et al., 2020).

Os mecanismos fisiopatológicos da covid-19 aguda são: infecção viral direta; lesão endotelial e dano microvascular; comprometimento do sistema imunológico e estimulação de um estado muito inflamatório; e má adaptação da via da enzima conversora de angiotensina II (NALBANDIAN A, et al., 2021).

A covid-19 é altamente infecciosa e todos os indivíduos infectados podem apresentar um quadro clínico leve ou desenvolver sintomas graves, como pneumonia e insuficiência respiratória aguda, podendo levar a óbito. Os indivíduos do grupo de maior risco para desfechos graves são os pacientes com doenças crônicas, imunossupressão, pacientes de meia-idade e idosos. Em relação aos fatores de riscos para morte hospitalar pelo novo coronavírus, foi detectado que $48 \%$ dos pacientes apresentavam comorbidades, sendo a hipertensão a mais comum (30\%). Como foi identificado uma alta prevalência de Hipertensão Arterial Sistêmica (HAS) em casos graves de covid-19, esta patologia tem sido considerada um fator de risco importante (SANTANA VVRS, et al., 2020).

Na Atenção Primária à Saúde (APS), a HAS é uma doença muito comum de ser encontrada sendo considerada um grande problema mundial de saúde pública (SANTIAGO ERC, et al., 2019). É uma condição clínica que apresenta inúmeros fatores causais, caracterizada por níveis elevados da pressão arterial. Em muitos casos, pode estar relacionada a alterações estruturais e/ou funcionais dos órgãos-alvos e alterações metabólicas (MELO LD, et al., 2020).

A covid-19 tem uma interação com o sistema cardiovascular aumentando os casos de enfermidade e óbito aos pacientes que cursam com HAS ou outras patologias do sistema cardíaco (SILVA WBH, et al., 2021). Grande parte dos infectados que precisam de internação por complicações sofrem de HAS (CÉSPEDES MEG, et al., 2020). 
Nos hipertensos, seu estado inflamatório está relacionado à disfunção endotelial, caracterizada por uma desproporção na produção de substâncias com ação vasoconstritora, principalmente, e vasodilatadora que leva a um estado de disfunção hemodinâmica de intensidade variável (CÉSPEDES MEG, et al., 2020).

O controle e a prevenção da HAS são constituídos por mudanças nos hábitos de vida como a realização de atividades físicas regular, dieta balanceada, evitar etilismo e tabagismo. A HAS quando não tratada e controlada adequadamente pode gerar uma série de complicações como infarto agudo do miocárdio, hemorragias e encefalopatia hipertensiva, cardiopatia isquêmica e insuficiência cardíaca (ARAÚJO GSB, et al., 2019)

Diante desse pressuposto, é importante entender qual a correlação entre a covid-19 e a HAS e porque essa comorbidade gera um pior prognóstico em pacientes infectados pelo coronavírus. Primeiro, para que sejam mais efetivos comportamentos que previnam a contaminação nesse grupo de risco, bem como otimizar a terapêutica da hipertensão a fim de evitar complicações na saúde dos pacientes (SANTIAGO ERC, et al., 2019).

Esse constructo teve como objetivo compreender a relação entre a covid-19 e a hipertensão arterial sistêmica.

\section{REVISÃO BIBLIOGRÁFICA}

A HAS é uma doença crônica definida por níveis pressóricos maiores ou iguais as 140/90 mmHg, representa um dos fatores de risco para doenças cardiovasculares. A HAS atinge cerca de 36 milhões de brasileiros, mais de $60 \%$ da população acima dos 60 anos e é responsável por $50 \%$ das mortes por doenças cardiovasculares. O mesmo ressalta que a prevalência da HAS no Brasil é de $30 \%$, sendo mais prevalente entre homens do que em mulheres (JUNIOR UESR e FERNANDES RCP, 2020).

Os fatores de risco da HAS podem justificar a sua alta prevalência. Esses podem ser não modificáveis (idade, gênero e etnia) e modificáveis (obesidade, alta ingesta de sal e de álcool, estresse, uso crônico de tabaco, sedentarismo e síndrome da apneia/hipopneia obstrutiva do sono) (CARDOSO FN, et al., 2020). Além disso, atualmente, a HAS é um dos principais fatores de risco para casos graves da covid-19 (SANTANA VVRS, et al., 2020).

A HAS é uma síndrome poligênica e apresenta aspectos ambientais, genéticos, vasculares, neurais e renais. Sobre os fatores ambientais, indivíduos que não têm hipertensão arterial e que se mudam para locais de alta prevalência de hipertensão, tornam-se hipertensos. O uso do sal tem importância na gênese da HAS em pessoas predispostas geneticamente. Os fatores vasculares são muito relevantes na fisiopatogenia pois a constrição funcional da musculatura lisa é considerada um dos principais mecanismos da HAS. Em relação aos fatores neurais, há evidências de que o sistema nervoso simpático é importante no aumento da gênese e na sustentação da HAS (NOBRE F, et al., 2013).

Quanto aos fatores renais, é bem definido que o aumento da pressão de perfusão arterial renal causa o aumento da excreção de sódio e água. Quando a pressão arterial aumenta, ocorre a ativação do fenômeno pressão-natriurese ocasionando a excreção de sódio e água até que ela seja reduzida a valores normais. Além disso, a causa da HAS na maioria das vezes (mais de 90\%) é desconhecida. Mas sabe-se que a ativação do sistema nervoso simpático, do sistema renina angiotensina aldosterona e a curva de pressão natriurese alterada é de grande importância para fisiopatogênese dessa patologia (LOPES HF, 2019).

A enzima renina, sob a ação de alterações na pressão da arteríola aferente renal e concentração de sódio na mácula densa, é liberada do aparelho justaglomerular e, quando interage com seu substrato plasmático angiotensinogênio, muda sua conformação para angiotensina I que por sua vez se transforma em angiotensina II. Essa propicia a vasoconstrição, eleva o tônus simpático, estimula a secreção de aldosterona e reabsorção de sódio e concorre para a liberação de hormônio antidiurético (NOBRE F, et al., 2013).

O diagnóstico da HAS é baseado no nível médio de pressão arterial maior ou igual 140/90 mmHg, aferidas no mínimo em três dias distintos com pelo menos sete dias de intervalo entre as medidas. A medição de um 
valor acentuadamente alto em apenas um dia, mesmo que em mais do que uma medida, não é o bastante para indicar o diagnóstico de hipertensão. Também são utilizados como métodos diagnósticos a Monitorização Ambulatorial da Pressão Arterial (MAPA) e a Monitorização Residencial da Pressão Arterial (MRPA) (BARROSO WKS, et al., 2021; BARROS GM, et al., 2020).

O uso de fármacos específicos receitados pelo médico, caracteriza o controle e tratamento medicamentoso dessa enfermidade silenciosa e progressiva. Medidas não medicamentosa, que estão relacionadas com a modificação do estilo de vida do paciente para comportamentos mais saudáveis, ajudam no controle da HAS. Quando não tratada de modo correto ou se não tratada, a hipertensão arterial pode levar a disfunção de alguns órgãos vitais (SILVA MLB, et al., 2016).

A covid-19 é causada pelo SARS-CoV-2, um betacoronavírus com ácido ribonucleico envelopado que tem uma proteína chamada spike protein ancorada no envelope viral. Essa proteína medeia a entrada do vírus nas células hospedeiras e se ligam ao receptor hospedeiro e depois se funde à membrana. Um domínio definido de ligação ao receptor do pico de SARS-CoV reconhece especificamente a Enzima Conversora de Angiotensina II (ECA II) que é expressa pelas células epiteliais do pulmão, intestino, rim e vasos sanguíneos, e assim, o vírus infecta a célula humana (ASTURIAN K, 2021).

A covid-19 é uma doença respiratória, identificada primeiramente na cidade de Wuhan, na China, que se espalhou pelo mundo, gerando um grande problema de saúde pública por sua rápida propagação. Embora possa apresentar um quadro clínico leve, a covid-19 pode desenvolver sintomas graves, como pneumonia e insuficiência respiratória aguda, podendo levar a óbito. Além disso, como possui um alto poder de transmissibilidade, o vírus pode gerar alta taxa de letalidade para grupos específicos como idosos, pessoas com comorbidades e imunodeficiência (NIQUINI RP, et al., 2021; OLIVEIRA WK, et al., 2020).

Além disso, foi observado o aumento da mortalidade e morbidade da covid-19 em pacientes com HAS. A maioria das mortes e complicações são em pacientes com 60 anos ou mais que apresentam alguma comorbidade. Em um estudo de coorte com 201 pacientes com pneumonia confirmada por covid-19, 84 deles desenvolveram síndrome do desconforto respiratório agudo, e destes 23 apresentaram a HAS como condição crônica mais comum nessa amostra (BARROS GM, et al., 2020)

As medidas de isolamento social no Brasil ocorreram devido uma alta propagação do vírus, a grande proporção de infectados oligossintomáticos ou assintomáticos que infectam pessoas saudáveis, a inexistência de terapia medicamentosa comprovada e a duração prolongada dos quadros clínicos (FILHO JMJ, et al., 2020).

O diagnóstico padrão ouro para a identificação do novo coronavírus é realizado por meio das técnicas de reação em cadeia da polimerase com transcrição reversa em tempo real (RT-PCR), e sequenciamento parcial ou total do genoma viral. As amostras para análise podem ser feitas pelo aspirado nasofaríngeo (ANF), swab nasal e oral e pela secreção respiratória do trato inferior. Apesar de ser o melhor teste diagnóstico, o resultado negativo do RT-PCR não descarta totalmente a possibilidade de infecção pelo vírus, por isso, é recomendado que o resultado seja avaliado conjuntamente com observações clínicas, o histórico do paciente e informações epidemiológicas (NOGUEIRA JMR e SILVA LOP, 2020).

A pandemia da covid-19 é ainda nova e sua duração é incerta. Portanto, reduzir a exposição ao vírus é necessário para controlar a propagação da doença e o índice de mortalidade. O distanciamento social é fundamental para diminuir a transmissão minimizando o contato entre indivíduos infectados e saudáveis. Dessa forma, a mudança de comportamento individual e coletivo nesse cenário é o caminho a ser seguido para o enfrentamento desse momento crítico (OLIVEIRA AC, et al., 2020).

O coronavírus adentra nas células por meio do receptor da ECA II. Paralelamente, pacientes portadores da HAS apresentam esses receptores em maior quantidade. Desse modo, estariam mais vulneráveis ao avanço do vírus nas células extrapulmonares (SILVA MS, et al., 2020).

No entanto, o receptor da proteína tirosina quinase, codificado pelo gene AXL interage especificamente com o domínio N-terminal do SARS-CoV-2. Foi demonstrado uma superexpressão de AXL em células 
HEK293T promovendo a entrada do vírus de forma tão eficaz quanto a superexpressão de ECA II. Assim, o coronavírus foi capaz de utilizar tanto a ECA Il quando AXL para a entrada em células humanas, sendo o AXL expresso em órgãos humanos principalmente em tecidos pulmonares e brônquicos, onde sua expressão é bem maior do que a expressão da ECA II (SOUZA DSM, et al., 2021).

O SARS-CoV-2 possui um envelope viral de glicoproteínas de pico compostas por duas subunidades (S1 e S2). A subunidade S1 se liga à superfície celular da ECA II e a subunidade S2 funde-se com a membrana celular. Por intermédio da proteinase TMPRSS2, então, ocorre a entrada do vírus na célula (SOUZA DSM, et al., 2021).

A ECA II faz parte do Sistema Renina Angiotensina Aldosterona (SRAA) que tem uma importante função como regulador da pressão arterial, da regulação de processos metabólicos e da homeostase eletrolítica renal. $O$ angiotensinogênio, produzido pelo fígado, é fracionado pela renina, dando resultado a formação de angiotensina I que posteriormente se transforma em angiotensina II pela catalização da ECA. Dentre vários efeitos, a angiotensina II tem como principais o aumento da pressão arterial e indução de vias inflamatórias e pró-fibróticas (SOUZA LV, et al., 2021).

Os dois principais receptores de angiotensina são: ATI 1s, presentes nos vasos sanguíneos renais, coração, glândulas adrenais, fígado e cérebro responsáveis pela maioria dos efeitos hipertensivos da angiotensina II, por isso, são um importante alvo terapêutico no tratamento de hipertensão e doenças cardiovasculares. Enquanto os receptores ATI 2 são encontrados no feto, ovários, útero, glândulas adrenais e vasculatura, portanto, apresentam influência nos efeitos vasodilatadores (GIRALT-HERRERA A, et al., 2020).

A ECA II não é só a porta de entrada do SARS-CoV-2, mas também medeia a lesão e insuficiência pulmonar. Essa, apresenta um papel protetor. A angiotensina 1-7, convertida pela ECA II a partir da angiotensina II, apresenta efeitos anti-apoptótico, anti-proliferativo, anti-fibrose e vasodilatador com vários efeitos protetores cardiovasculares. A diminuição da atividade da ECA II no coração devido a infecção do coronavírus, favorece o aumento de angiotensina II e expressão da ECA I causando aumento da vasoconstrição, sobrecarga do coração e alta pressão arterial (MARTINS JDN, et al., 2020).

Existe o questionamento se pacientes com covid-19 e hipertensos em tratamento com Inibidores da Enzima Conversora de Angiotensina (IECA) e Bloqueadores dos Receptores de Angiotensina (BRA) devem ou não consumir esses medicamentos (CÉSPEDES MEG, et al., 2020). Ambos têm a função de aumentar a expressão da ECA II, bloqueando a via da ECA I e consequentemente a síntese de angiotensina II, diminuindo a pressão sanguínea, inflamação e fibrose (VIEIRA MS, et al., 2021).

Em Whuan, foi realizado um estudo comparativo entre pacientes com covid-19 que fazem uso de IECA/BRA e um grupo que fazia tratamento com outro grupo de anti-hipertensivo. Foi observado que os que usavam IECA/BRA apresentaram uma queda tanto de proteína $C$ reativa de alta sensibilidade, quanto nos níveis do biomarcador procalcitonina (PCT). Assim, foi possível concluir que o uso de IECA/BRA não se caracteriza como risco adicional para infecção por covid-19 quando comparado ao uso de outros medicamentos anti-hipertensivos (YANG G, et al., 2020).

Além disso, hipertensos com covid-19 em que o uso hospitalar do IECA/BRA esteve associado a menores taxas de mortalidade em comparação aos que não utilizaram IECA/BRA. Esses achados sugerem que o uso continuo desses medicamentos devem ser mantidos para controlar a pressão arterial tendo como objetivo 0 benefício do paciente (DENG Y, et al., 2021).

Pacientes hipertensos com covid-19 que faziam uso do IECA/BRA, foi observado que o aparecimento dos sintomas relacionados à infecção só ocorreu após 20 dias, em comparação ao aparecimento em 16 dias daqueles que não utilizavam IECA/BRA. Por conseguinte, em um estudo de caso de um paciente de 41 anos, hipertenso e com covid-19, em uso de BRA, teve seu anti-hipertensivo substituído por antagonistas de cálcio e após essa troca percebeu-se uma desordem da via do receptor ECA II, resultando no desequilibro do SRAA e agravamento da inflamação pulmonar (SOUZA SS, et al., 2021). 
Apesar de pacientes infectados terem uma expressão mais elevada de ECA II e isso estar relacionado com cargas virais mais altas, os IECAs/BRAs não deveriam ser descontinuados, pois têm um efeito protetor contra lesões cardíacas e reduzem a gravidade da doença pulmonar. No entanto, os pesquisadores não acham necessário fazer o uso imediato dos fármacos, por não existirem evidências definitivas de que eles beneficiem a sobrevida dos pacientes infectados por SARS-CoV-2 (SOUZA SS, et al., 2021).

No estudo de Meng J, et al. (2020), foram selecionados 42 pacientes divididos em dois grupos com base em terapia anti-hipertensivas. O primeiro grupo foi tratado com medicamentos IECA/BRA e o segundo grupo recebeu tratamento com outros anti-hipertensivos como betabloqueadores e diuréticos. Foi observado que 0 primeiro grupo apresentou menor taxa de doenças grave, tendência a menor nível de interleucina-6 (IL-6) no sangue periférico, aumentou a contagem de células TCD3 e CD8 no sangue periférico e diminuiu o pico de carga viral em comparação ao segundo grupo. Além disso, ao se comparar o uso de IECA ou BRA para pacientes com covid-19, o uso do BRA mostrou ser mais auspicioso devido a menos efeitos colaterais, angioedema e tosse pelo IECA (VIEIRA MS, et al., 2021).

A quantidade de linfócitos em pacientes com HAS é outra evidência da correlação com o SARS-CoV-2. Estudos mostram que pacientes portadores de HAS apresentam baixas contagens de linfócitos quando comparados aos não hipertensos, o que gera a sugestão de que essa redução é ocasionada pela redução de ECA II gerada a partir da invasão do vírus da covid-19 (SILVA MS, et al., 2020). A linfopenia também é uma característica dos pacientes com covid-19, principalmente em casos graves. Os pacientes também apresentam uma queda acentuada no número de células TCD4+, TCD8+, NK e B (YANG L, et al., 2020).

Um estudo realizado com 337 pacientes hospitalizados com covid-19 mostrou que desses, 122 (33,2\%) eram hipertensos, sendo a idade média de 58 anos e 50\% dos pacientes eram do sexo masculino. Essa pesquisa ratificou que além da linfopenia, pacientes hipertensos e infectados pelo coronavírus apresentaram contagens altas de leucócitos, neutrófilos e níveis elevados de triglicérides e bilirrubina. Demonstraram também que o estágio da hipertensão esteve associado à gravidade da doença e ao resultado clinico. (DENG Y, et al., 2021). Ademais, foi analisado que o grupo de hipertensos mostrou uma relação um pouco maior de infecções bacterianas, sem significância estatística, mas que levou a uma maior chance de mortalidade (DENG Y, et al., 2021).

\section{CONSIDERAÇÕES FINAIS}

Baseado em todas as evidências cientificas apresentadas nessa revisão de literatura, a hipertensão arterial tem associação significativa com a gravidade e mortalidade da covid-19. Os pacientes hipertensos e infectados pelo coronavírus podem apresentar severas manifestações clinicas e complicações em órgãosalvos. Isso se deve à ligação entre o vírus e o receptor ECA II, e outro destaque é o baixo nível de linfócitos em hipertensos. Ademais, os estudos mostram que o tratamento anti-hipertensivo parece não agravar a infecção pelo SARS-CoV-2. O uso dos fármacos anti-hipertensivos, por hora, não deve ser interrompido, pois as evidências atuais não são consistentes quando relacionam a terapia com o risco de má evolução pela covid-19. Ainda não existe uma terapia especifica para pacientes infectados pelo vírus, então medicações que podem contribuir a intensificar a imunidade do paciente de forma favorável é de extrema importância.

\section{REFERÊNCIAS}

1. ARAÚJO GSB, et al. Hipertensão arterial sistêmica: problema de saúde pública nos dias atuais. Revista Brasileira Interdisciplinar de Saúde, 2019; 1(1): 39-43.

2. ASTURIAN K. O papel dos inibidores da enzima conversora de angiotensina e dos antagonistas dos receptores de angiotensina em pacientes com Covid-19: uma revisão narrativa. Revista de Ciências Médicas, 2021; 30 : e215035

3. BARROS GM, et al. Considerações sobre a relação entre a hipertensão e o prognóstico da COVID-19. Journal of Health \& Biological Sciences, 2020; 8(1): 1-3.

4. BARROSO WKS, et al. Diretrizes Brasileiras de Hipertensão Arterial-2020. Arquivos Brasileiros de Cardiologia, 2021; 166: $516-658$

5. CARDOSO FN, et al. Fatores de risco cardiovascular modificáveis em pacientes com hipertensão arterial sistêmica. Revista Mineira de Enfermagem, 2020; 24: e1275. 
6. CÉSPEDES MEG, et al. La COVID-19 en personas hipertensas. Medisan, 2020; 24(3): 501-514.

7. DENG Y, et al. Associação da Hipertensão com a Gravidade e a Mortalidade de Pacientes Hospitalizados com COVID19 em Wuhan, China: Estudo Unicêntrico e Retrospectivo. Arquivos Brasileiros de Cardiologia, 2021: 1-11.

8. FILHO JMJ, et al. A saúde do trabalhador e o enfrentamento da COVID-19. Revista Brasileira de Saúde Ocupacional, São Paulo, 2020; 45: e14.

9. GIRALT-HERRERA A, et al. Relación entre COVID-19 e hipertensión arterial. Revista Habanera de Ciencias Médicas, 2020; 19(2): 1-11.

10. JUNIOR UESR, FERNANDES RCP. Hipertensão arterial em trabalhadores: o efeito cumulativo das dimensões da atividade física sobre esse agravo. Arquivos Brasileiros de Cardiologia, 2020; 114(5): 755-761.

11. LIMA AR, et al. Caracterização epidemiológica dos casos de covid-19 no mundo e brasil. Revista Cathedral, 2020; 2(4): 61-73.

12. LOPES HF. Hipertensão Arterial: Aspectos Fisiopatológicos, Estresse Psicossocial e Preferência por Alimentos. Arquivos Brasileiros de Cardiologia, 2019; 113(3): 381-382.

13. MARTINS JDN, et al. As implicações da COVID-19 no sistema cardiovascular: prognóstico e intercorrências. Journal of Health e Biological Sciences, 2020; 8(1): 1-9.

14. MELO LD, et al. Tabagismo, Hipertensão Arterial Sistêmica e Pandemia da COVID-19: uma Análise Psicanalítica Freudiana. Research, Society and Development, 2020; 9(11): e57891110240.

15. MENG J, et al. Os inibidores do sistema renina-angiotensina melhoram os resultados clínicos de pacientes com COVID-19 com hipertensão. Micróbios emergentes e infecções, 2020; 9(1): 757-760.

16. NALBANDIAN A, et al. Síndrome pós-aguda de COVID-19. Nature medicine, 2021; 27(4): 601-615.

17. NIQUINI RP, et al. SRAG por COVID-19 no Brasil: descrição e comparação de características demográficas e comorbidades com SRAG por influenza e com a população geral. Cadernos de Saúde Pública, 2020; 36: e00149420.

18. NOBRE F, et al. Hipertensão arterial sistêmica primária. Medicina, Ribeirão Preto, 2013; 46(3): $256-272$.

19. NOGUEIRA JMR, SILVA LOP. Diagnóstico laboratorial da COVID-19 no Brasil. Revista Brasileira de Análises Clínicas, 2020; 52(2): 117-121.

20. OLIVEIRA AC, et al. O que a pandemia da covid-19 tem nos ensinado sobre adoção de medidas de precaução? Texto e Contexto-Enfermagem, Florianópolis, 2020; 29: e20200106.

21. OLIVEIRA WK, et al. Como o Brasil pode deter a COVID-19. Epidemiologia e Serviços de Saúde, 2020; 29(2): e2020044.

22. SANTANA VVRS, et al. Fatores de Risco Para o Agravamento da COVID-19 em Indivíduos Jovens. Enfermagem em foco, 2020; 11(2): 37-45.

23. SANTIAGO ERC, et al. Prevalência e fatores associados à hipertensão arterial sistêmica em adultos do sertão de Pernambuco, Brasil. Arquivos Brasileiros de Cardiologia, São Paulo, 2019; 113(4): 687-695.

24. SILVA MLB, et al. Representações sociais da hipertensão arterial. Temas em Psicologia, 2016; 24(3): 895-909.

25. SILVA MS, et al. Evidências científicas clínicas entre o novo coronavírus e a hipertensão arterial: uma revisão integrativa. Journal Nursing Health, 2020; 10(4): e20104035.

26. SILVA WBH, et al. O que a pandemia da COVID-19 fez com a pressão arterial sistêmica? Global Academic Nursing Journal, 2021; 2(1): e105.

27. SOUZA DSM, et al. Na covid-19 é real a não suspensão dos inibidores do sistema renina angiotensina aldosterona? Revista Brasileira de Hipertensão, 2021; 28(1): 20-6.

28. SOUZA LV, et al. Inibidores da enzima conversora de angiotensina são fatores de risco ou proteção na COVID-19? Multidisciplinary Reviews, 2021; 4: e2021017.

29. SOUZA SS, et al. Influência da cobertura da atenção básica no enfrentamento da COVID-19. Jornal Health NPEPS, $2021 ; 6(1): 1-21$.

30. STRABELLI TMV, UVERSON D. COVID-19 e o Coração. Arquivos Brasileiros de Cardiologia, 2020; $114(4): 598-600$.

31. VELAVAN TP, MEYER CG. The COVID-19 epidemic. Medicina tropical e saúde internacional, 2020; 25(3): 278-280.

32. VIEIRA MS, et al. O uso de inibidores do sistema renina angiotensina aldosterona em pacientes hipertensos com COVID-19. Revista Eletrônica Acervo Saúde, 2021; 13(10): e8779.

33. YANG G, et al. Effects of angiotensin II receptor blockers and ACE (angiotensin-converting enzyme) inhibitors on virus infection, inflammatory status, and clinical outcomes in patients with COVID-19 and hypertension: a single-center retrospective study. Hypertension, 2020; 76(1): 51-58.

34. YANG L, et al. COVID-19: immunopathogenesis and Immunotherapeutics. Transdução de sinal e terapia direcionada, 2020; 5(1): 1-8.

35. WU Y, et al. O surto de COVID-19: uma visão geral. Jornal da associação médica chinesa, 2020; 83(3): 217. 